\title{
Study on an Adaptive Coding and Decoding Scheme on Distributed Space-Frequency coding

\author{
Li Yu ${ }^{1, a}$, Hou Zemin ${ }^{2, b}$, Zhang Bao-long ${ }^{2, c}$ \\ Zhengzhou,Henan,450064,China \\ ${ }^{2}$ College of Information Engineering , Zhengzhou University of Science and Technology, \\ Zhengzhou,Henan,450064,China

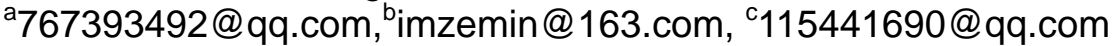 \\ ${ }^{1}$ College of Electrical Engineering , Zhengzhou University of Science and Technology,
}

\begin{abstract}
Keywords: Cooperative Communication; Distributed Linear Dispersion Codes; Space-frequency coding; condition number; Adaptive
\end{abstract}

\begin{abstract}
A new adaptive coding and decoding scheme is proposed for distributed space-frequency coding system. Each relay node selects dispersion matrix and modulation type adaptively according to channel state and different detection methods are used according to the threshold of channel condition number adaptively. The MATLAB simulation is performed under COST207 typical urban channel model. Simulation results show that the proposed adaptive coding scheme gives a more good performance.
\end{abstract}

\section{Perface}

The combination of MIMO and OFDM provides a new direction of wireless communication ${ }^{[1]}$. However, it is hard to install multiple antennas on mobile devices because of the limitation in size, power or hardware complexity. Cooperative diversity system has been proposed to overcome the difficulty. A virtual MIMO system is constituted by sharing each other's single antenna on different mobile device ${ }^{[2-4]}$.

Among the existing space-time codes, the linear dispersion code (LDC) is often preferred. This is because it subsumes many existing block codes as its special cases, allowing suboptimal linear receivers with greatly reduced complexity, and providing a flexible rate-versus-performance tradeoff $^{[5-6]}$. Among various kinds of detection algorithm, maximum likelihood (ML) detection is the optimal algorithm, but the decoding complexity increases exponentially with the increase of antenna number and modulation order. Linear detection algorithms such as ZF and MMSE, the complexity are lower but the performance has a big gap compared with $\mathrm{ML}^{[7]}$. Channel matrix condition number has an important influence on channel capacity. It is showed that the performance differences between suboptimal detection algorithms with ML algorithm performance is rooted in the influence of the condition number.

A new adaptive coding and decoding scheme is proposed for distributed space-frequency coding system. Each relay node selects dispersion matrix and modulation type adaptively according to channel state and different detection methods are used according to the threshold of channel condition number adaptively. The MATLAB simulation is performed under COST207 typical urban channel model. Simulation results show that the proposed adaptive coding scheme gives a more good performance.

\section{System model}

System model is shown in Fig.1. One source node and receive node, relay node have M. The source node and relay node have only one antenna while the number of receive antenna is $N$. We assume that all the nodes are ideal synchronizing. The subcarrier number of OFDM is $K$. Source node will sent modulated data to the relay node, Relay nodes use space-frequency coding and launch out with the OFDM modulation. The receiver demodulate with OFDM when getting the send information. 


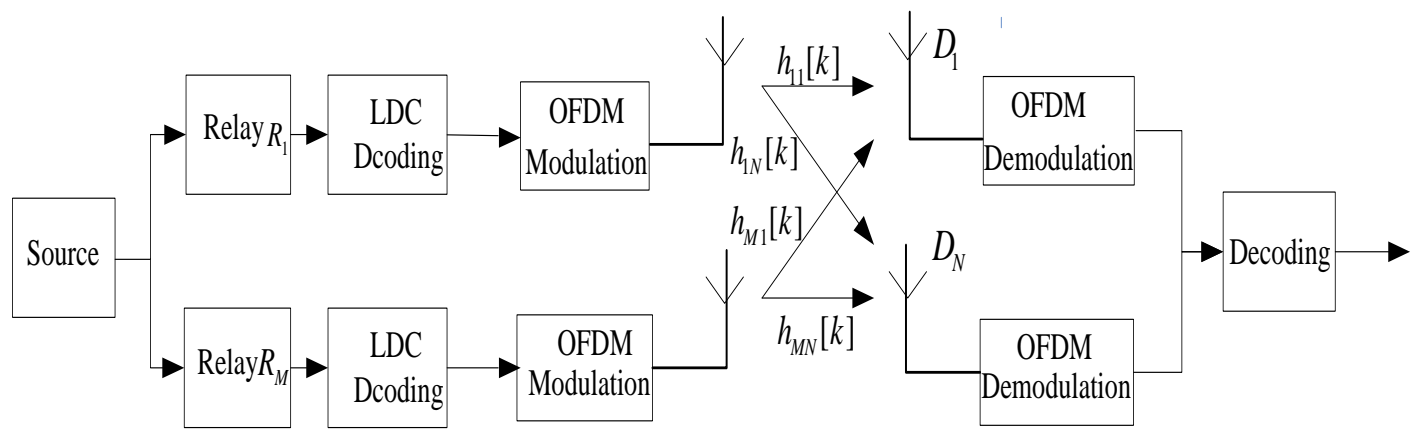

Fig.1 System model of Space-Frequency coding

When then length of $\mathrm{CP}$ is greater than the multipath time delays, the system can completely eliminate intersymbol interference (ISI) and interchannel interference (ICI). After the removing of $\mathrm{CP}$ and FFT computation, the received symbol of Kth subcarrier can be represented as:

$\boldsymbol{r}[\boldsymbol{k}]=H[k] \boldsymbol{x}[\boldsymbol{k}]+\boldsymbol{n}[\boldsymbol{k}], \quad k=1,2, \cdots, K$

( 1 ) Where $\boldsymbol{x}[\boldsymbol{k}]=\left[x_{1}(k), x_{2}(k), \cdots, x_{R_{M}}(k)\right]^{T}$ is the sending vector symbols of Kth carrier of each relay node, $\boldsymbol{n}[\boldsymbol{k}]=\left[n_{1}(k), n_{2}(k), \cdots, n_{D_{N}}(k)\right]^{T}$ is White gaussian noise, $H[k]=\left[\begin{array}{ccc}h_{1,1}[k] & \cdots & h_{1, N}[k] \\ \vdots & \ddots & \vdots \\ h_{M, 1}[k] & \cdots & h_{M, N}[k]\end{array}\right]$ is Frequency domain channel matrix.

Assuming the system is completely synchronized. Source node, relay node and the receiver can obtain the ideal channel information, the system can be viewed as a virtual MIMO system, the receiver can adopt MIMO system detection algorithm for decoding.

\section{Distributed linear dispersion codes (LDC)}

LDC is defined by[5]:

$$
S=\sum_{q=1}^{Q}\left(\alpha_{q} A_{q}+j \beta_{q} B_{q}\right)
$$

Among $\mathrm{Q}$ is send information symbol for each group, $s=\left[s_{1}, s_{2}, \cdots, s_{Q}\right]^{T} . \alpha_{q}, \beta_{q}$ is the real part and imaginary part of the first $q$ symbol . The key to LDC design is the duration of a set of symbols $T$, the number of information symbol $Q$, the dispersion matrix $A_{q}$ and $B_{q}$. Through the set of parameters, LDC can compromise between diversity gain and multiplexing gain. The specific design process are detail introduced in the literature [5] [6].

In cooperative communication space-frequency coding system, coding is in the spatial domain and frequency domain. The sending time is Q symbol period in the first phase, the duration of the second phase of symbols embodied in occupying the subcarrier number, set to $\mathrm{N}$. The transmission speed of the system is redefined as

$$
R=\left(\frac{Q}{Q+N}\right) \log _{2} r
$$

Where $r$ is modulation order (such as $r-Q A M, r-P S K$ ).

\section{Adaptive space-frequency coding and decoding scheme}

The condition number of Channel matrix $H$ is defined as

$$
c_{H}=\frac{\lambda_{\max }}{\lambda_{\min }}
$$


Among them, $\lambda_{\max }$ and $\lambda_{\min }$ is the maximum and minimum singular value of matrix H. Matrix condition number reflects the degree of morbid about equations, plays an important role in the MIMO system. Literature [8] showed, the performance difference between suboptimal detection algorithm from ML algorithm performance lies in the influence of the condition number. Condition number describes the distortion degree of the multidimensional constellation [8], the greater the condition number, the higher the level of distortion, the greater influence of noise on emission signal, signal decoding will become more difficult. The condition number of Channel matrix more tend to 1 , the greater the channel capacity, the linear detection can also get the same diversity order as maximum likelihood detection [8]. According to condition number and channel state, the adaptive coding and decoding scheme can be designed as follows:

(1)Relay nodes select coding scheme adaptively

Signal-to-noise ratio is one of the key indicators to measure the channel state, different linear dispersion codes have different performance under the same SNR. As shown in fig2. $T=2, Q=4,8 P S K$ and $T=2, Q=2,16 Q A M$ have the same code rate. When $S N R<10 d B \quad, \quad T=2, Q=4,8 P S K$ performance better than $T=2, Q=2,16 Q A M$. When $S N R>10 d B$, the latter performance is better. So we can choose different linear dispersion codes according to the current channel SNR to improve the performance of the system.

(2)The receiver select decoding scheme adaptively

The condition number can be calculated by $C_{H}=\|H\|^{*}\left\|H^{-1}\right\|$, Setting the threshold value of $\theta$ according to $C_{H}$. If $C_{H}<\theta$ use MMSE decoding else use ML decoding. The more $C_{H}$ close to 1 , the more linear detection performance close to the detection of maximum likelihood. Due to the lower complexity of the linear detection than ML decoding, it can adjust the size of the $\theta$ to weigh the decoding complexity and system bit error probability.

\section{Performance Simulation}

Relay node number is 2 , the receiver antenna number is 2 , the channel from source node to relay node is flat Rayleigh fading, the channel from Relay node to the destination node is COST207 typical urban channel model of 6 diameter. Relay node and destination node can obtain the ideal channel information. OFDM sub-carrier number is 256, $10 \mathrm{MHz}$ bandwidth, Each frame own 40 bit cyclic prefix. Multipath fading and multipath delay of channel is as follows:

$$
\begin{aligned}
& \text { Multipath fading }=\left[\begin{array}{llllll}
0.4 & 0.541 & 0.383 & 0.306 & 0.43 & 0.342
\end{array}\right] \\
& \text { Multipath delay }(\mu S)=\left[\begin{array}{llllll}
0.0 & 0.3 & 1.0 & 1.6 & 5.0 & 6.6
\end{array}\right]
\end{aligned}
$$

Fig.2 shows the BER performance of $T=2, Q=2,16 Q A M$ and $T=2, Q=4,8 P S K$ in the distributed space-frequency coding using ML decoding. These two kinds of coding scheme have the same rate. It can be seen that the two coding scheme have different bit error rate performance in different SNR range. So it is necessary to use different encoding mode and modulation mode to improve the performance according to the different channel state .

Fig. 3 simulated the adaptive decoding scheme which proposed In this paper, linear dispersion codes use $T=2, Q=4,8 P S K$, the decoding condition threshold is 3,5 and 7 . The simulation results show that the smaller the threshold value, the better the decoding performance. But with the decrease of the threshold value, It will be more likely to use ML decoding, the complexity of decoding will increased accordingly.

Fig.4 combine adaptive coding with decoding, the threshold of decoding condition number is $\theta=3$. Candidate code selects the codes which used in figure 2, threshold of adaptive SNR set to $10 \mathrm{~dB}$. It can be found that performance of the system is further improved after joining the adaptive coding. The adaptive coding will select low-order modulation when channel condition is bad, to a certain extent, it also reduces the decoding complexity. 


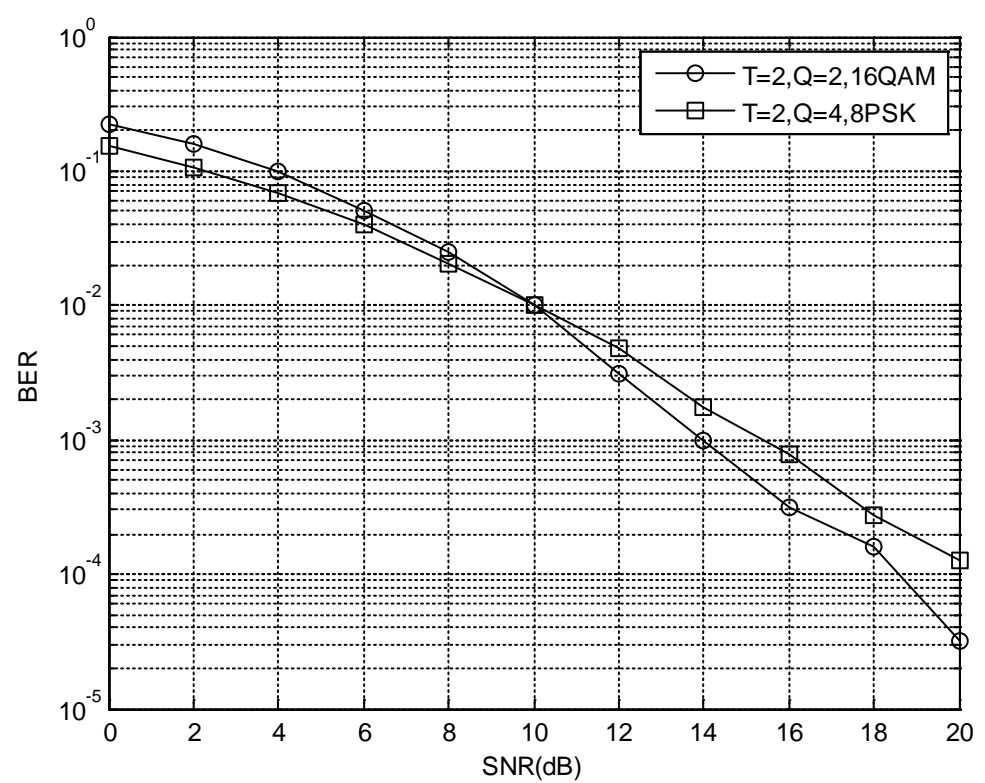

Fig.2 Performance comparison of LDC have same code rate but different dispersion matrix

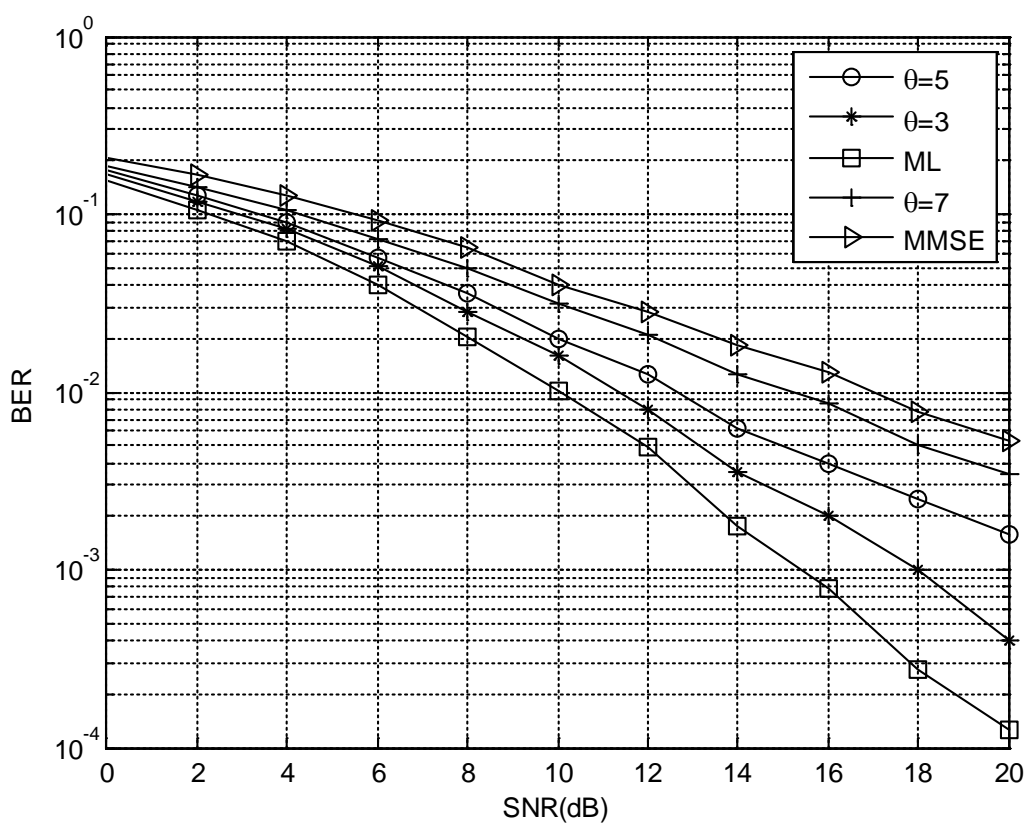

Fig.3 Performance of adaptive decoding $\mathrm{T}=2, \mathrm{Q}=4,8 \mathrm{PSK}$ 


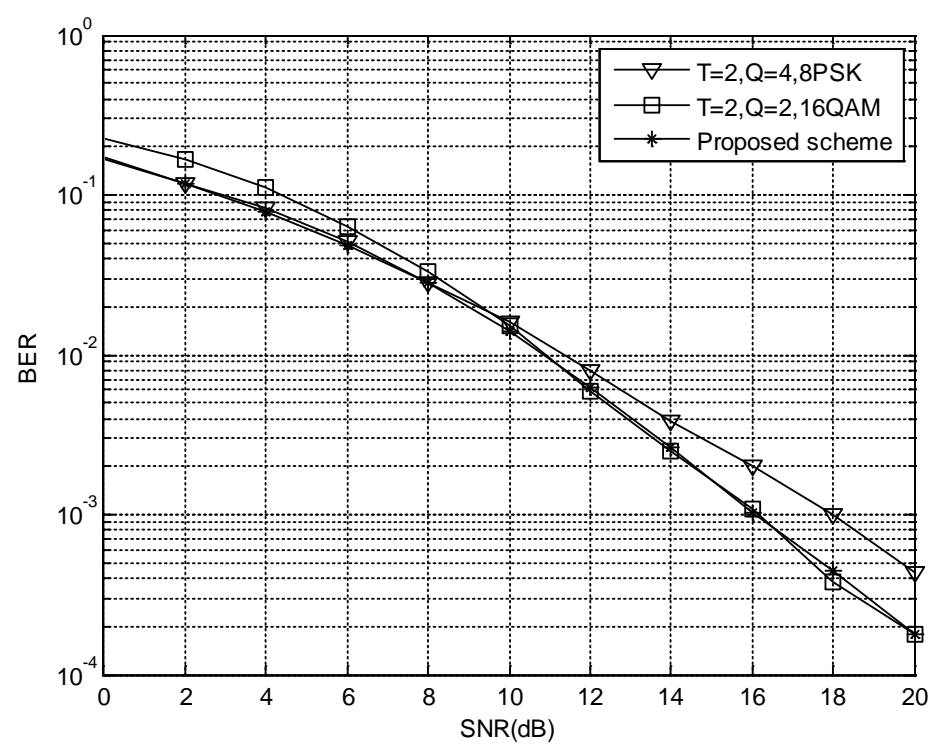

Fig.4 Performance of adaptive coding and decoding scheme

\section{Summary}

A novel adaptive coding and decoding scheme for DLDC-OFDM system is proposed, making full use of the channel condition number. Each relay node selects dispersion matrix and modulation type according to the channel state, and different detection methods are used according to the threshold of channel condition number adaptively. The MATLAB simulation is performed under COST207 typical urban channel model. Simulation results show that the proposed adaptive coding scheme gives a more good performance.

\section{References}

[1] Zhou En,Zhang Guang,Lv ZhaoBiao. The next generation broadband wireless communication OFDM with MIMO technology[M].BeiJing:People's posts and telecommunications publishing house,2008.

[2] Y. Zhang, H.-H. Chen, and M. Guizani. Cooperative Wireless Communications[M]. CRC Press, 2009.

[3] Mischa Dohler, Yonghui Li. Cooperative Communications: Hardware, Channel \& PHY[M]. John Wiley and Sons Press, 2010

[4]Xiao XiaoChao,Zheng BaoYu,Xu XiaoRong. Research of distributed Space-Time Coding techniques in Co-MIMO system[J].SINNAL PROCESSING, 2011,27(3): 340-345.

[5]Babak Hassibi and B.M. Hochwald, High-Rate Codes That Are Linear in Space and Time[J]. IEEE Transactions on Information Theory, July 2002, 48(7): 1804-1825.

[6] Ramy H. Gohary and Timothy N. Davidson. Design of Linear Dispersion Codes: Asymptotic Guidelines and Their Implementation[J]. IEEE Transactions on Wireless Communications, Nov 2005, 4(6): 2892-2906.

[7] Xiang YuLing. Multiple antenna signal launch plan and testing technology research in the system [D].Chengdu:University Of Electronic Scinence And Technology Of China,2008.

[8]Johannes Maurer, Gerald Matz, Dominik Seethaler. Low-complexity and Full-diversity MIMO Detection Based ON Conditon Number Thredholdinn. IEEE ICASSP, 2007 : 61 64. 